\title{
Creating Image Mosaics using Statistical Methods for Images Alignment
}

\section{Israa Mohammad Khidher Mohammed A. M. Al-taee \\ Department of Computer Science / Collage of Education \\ Mosul University}

Received

22 / 12 / 2008
Accepted

05 / 05 / 2009

الخالصة

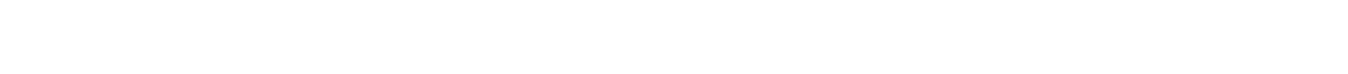

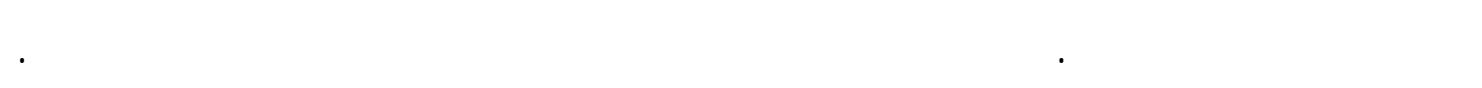

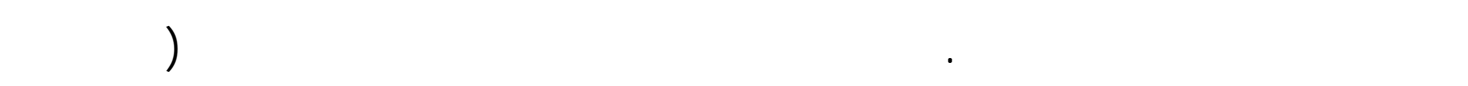

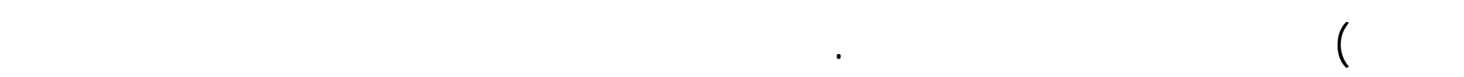

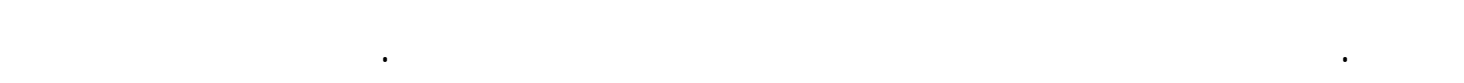

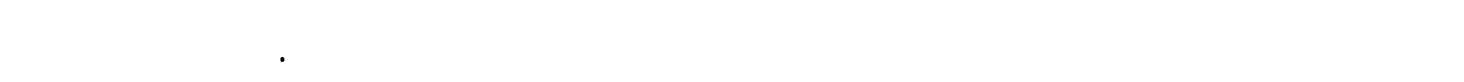
بناء فسفساء الصور باعتماد الطرائق الإصائية.

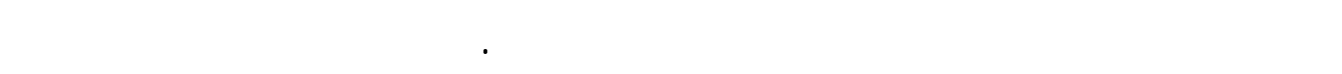

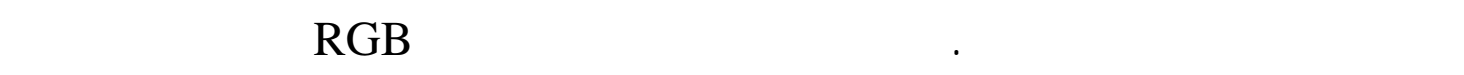

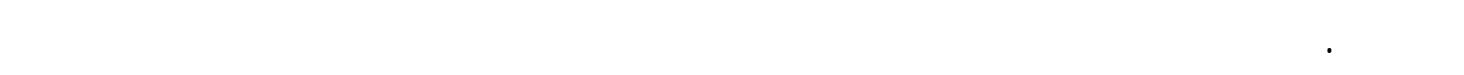

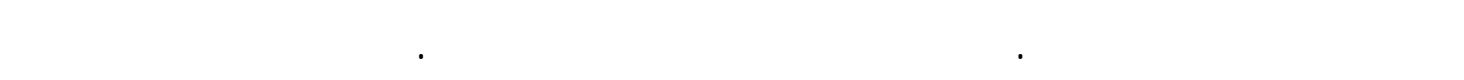

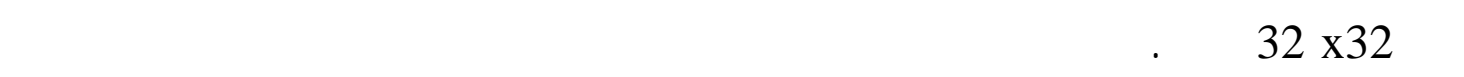

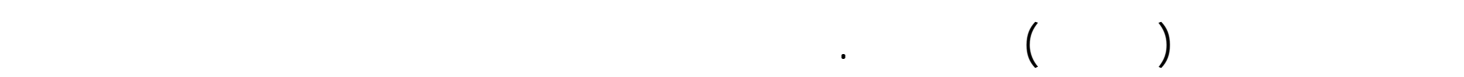

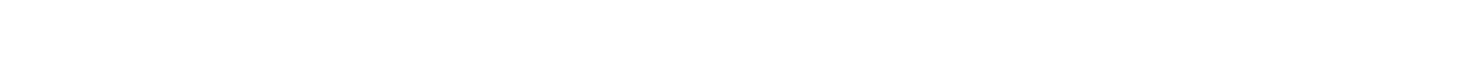

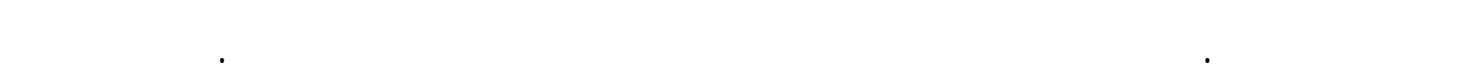

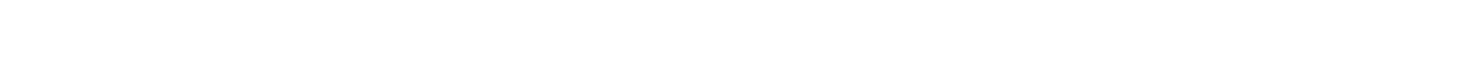

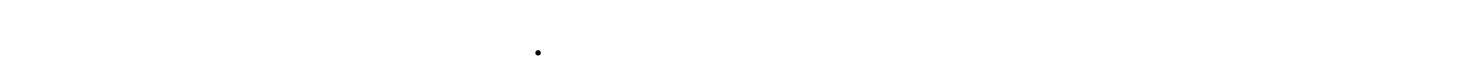

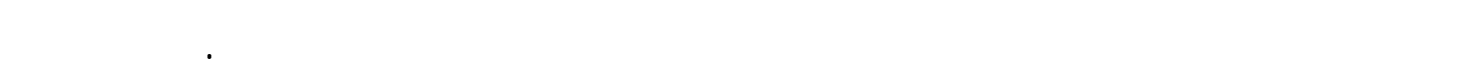

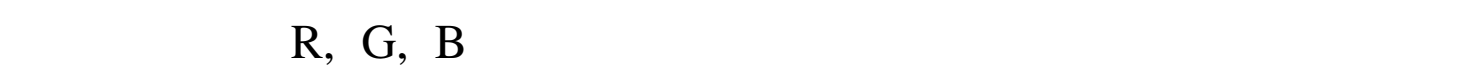

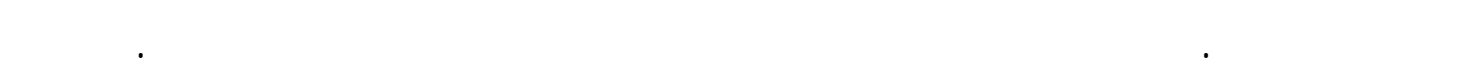
لذلك تمت بناء فسيفاء الصورة. عرض العطل المقترح نتائج دقيقة وكفؤ. 


\section{Abstract}

Image mosaics is a collection of small images arranged in a way that when they are seen from a distance they seen as larger image. It occupies a wide field in computer vision and image processing area. This field is performed in four stages. These stages are selecting the target image (input image) stage, choosing the tiling pattern stage. These tiles are small images from image database. The third stage arranged the tiles in a way to cover the target image. Indeed, color correction stage where the image colors are adjusted to better match the target image colors. The aim of our work is to create an image mosaics based on Statistical approaches.

Database images are collected and selected randomly. Then these images are pr-processed through a resized operation. Also a color transformation from RGB to the grayscale form (intensity). The color transformation is necessary because the intensity form shows the details of the target and the tile images to obtain effective images matching. At first we select a target image randomly. The target image is segmented to $32 \times 32$ tiles. At the second stage we choose the rectangular pattern for its simplicity as well as the rectangular nature of tile images used. Arranging tile stage is obtained, for each tile the system search the image database and selects the best matching image based on correlation coefficients of the intensity images. At this stage the difference between our works than others appeared. We use the correlation coefficients for matching operation that obtain a best matching than other approaches like Mathematical approaches which obtained approximate results such as distance functions. Also other Statistical approaches such as Histogram intersection and Statistics $\aleph^{2}$ measure (Chi Square) used to arrange tiles operation. Finally, we perform image color correction in pixel wise using the average of the $\mathrm{R}, \mathrm{G}, \mathrm{B}$ channels of the target and tile images. The resultant pixel obtained from last step will cover the target. As a result an image mosaic is created. The proposed work shows efficient and accurate results.

\section{Introduction}

Image mosaics is a composition of a small images called "tiles". In order to reproduce the mosaics - like style these tiles are covered on a target image. These tiles are images selected either randomly or automatically from image database and scaled as a thumbnail. An image mosaic is regarded as an active research area from two important fields. The first field is the computer vision while, the second field is the computer graphics [1]. In computer vision image mosaics is a huge area from a known study named visual scene representation. The scene representation deals with a collection of scene [2]. Furthermore, in 
computer graphics image mosaic represents image based rendering which attempt to create mosaics from a collection of images [1].

The rapid progress in image processing and computer vision in the last decades affects image mosaics area and appeared in its various applications [6]. The usefulness of this application appeared clearly in GIS (geographic information system) and remote sensing as large aerial and satellite photographs. Recently, image mosaic is used in scene stabilization, change detection, image compression and video indexing. Also it increases the view and resolution of camera and photo editing $[7]$.

There exist various types of image mosaic such as periodic or nonperiodic image mosaics. Periodic image mosaic is created by one image covered overall the target image, but the non-periodic used more than one image to cover the target image [2].

\section{Review of Previous Work}

Researchers have produced a rich literature in image mosaics area. Yue Zhang refer to a historic age of image mosaics have been started since $16^{\text {th }}$ century by a painter Giuseppe Aramboldo, he used plants and flowers to invent image mosaics. A graduate student from Massachusetts Information of Technology Lab invents a big progress in computer vision by generating an automatic image mosaics system known by "photo mosaics" [10]. Di Blasi Gianpiero et.al, refer to a work known by photo mosaic transform a target image to small grid and covered with thumbnails dataset images that approximate the pixels grid. Another work by Haeberli described a method and used Voroni diagrams to create a given picture, but composed of tiny brush strokes. Merrier used the same idea but with 3D, Lit Winowicz extended his idea to video [1].

Yue Zhang said that Echen uses different colors for his different letters forming his "Art from text". A work by Marius Hartman used different faces to compose facial image [10]. Yue Zhang refer to a work by Hausner used centroidal Voroni diagrams edge features to optimize the results he used graphic acceleration hardware [1].

Di Blasi Gianpiero et., al noted to a research by Chiba et., al, extract eight features varies between color, texture and shape then divided the target image into a triangles shape covered with the thumbnails images. Indeed, Kim and Pellacini introduced a picture by image tiles of arbitrary shape [1]. Our work composed of four stages. These stages are: input the target image, choosing the tiling pattern, arranging tiles represented by image database which covered the target image. At this stage the matching operation is necessary our work is based on correlation coefficient to perform matching as a Statistical approach from Mathematics. But others widely used distance functions such as Euclidian distance and the Histogram intersection for matching. Indeed, the color correction stage. 
Creating Image Mosaics using Statistical Methods for Images Alignment.

\section{Creating image Mosaics}

In this section we introduce how to create an image mosaics. Specifically, given a collection of dataset images that is resized as thumbnail and covered a target image as shown in Figure (1) [10]. Ti and a target image I. The mosaics are created by covering image I with tiling images Ti's [2] . This process required 4 steps:

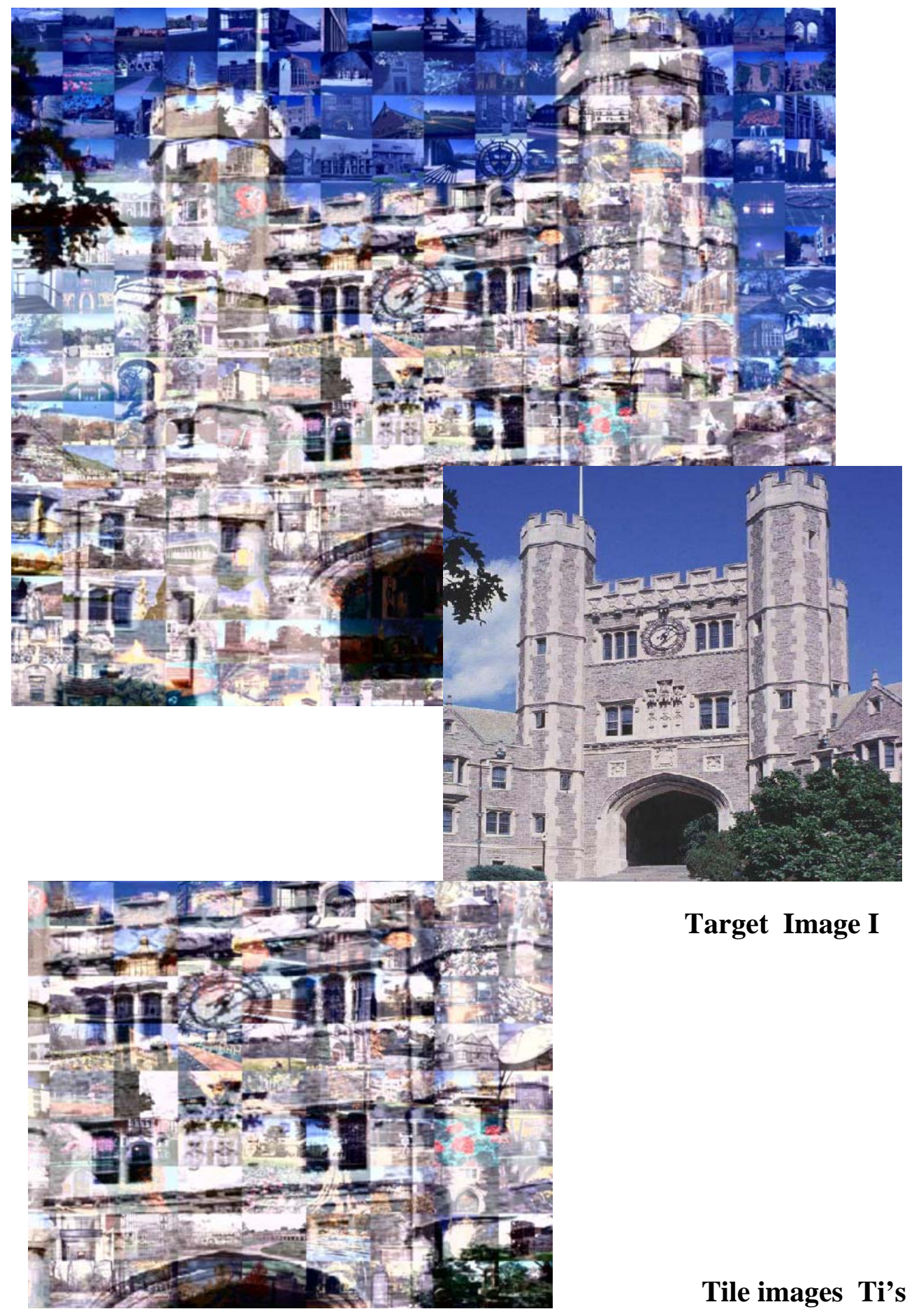

Figure (1): Image mosaics 


\subsection{Choosing Images}

The choice of target image is an important subject; this choice is an artistic matter. It depends on a verity factors. These factors are:

Recognition is an important factor, where the target and the tile figures with famous scenes could be commonly desirable such as historic leaders, work of arts, painters and actors. These famous scenes give more beauty to the mosaics creation. Other factors also such as color distribution in the image, as well as the brightness distribution which make the color correction easier [2][9].

\subsection{Defining Tiling}

A number of tiling patterns are exists for appropriate mosaic arrangement these patterns are the regular, the irregular, the semi-regular horizontal, and the semi-regular vertical as shown in Figure (2). Figure (3) shows a grayscale tiling images as tiling patterns [3]. The regular model is commonly used for it is simplicity and efficiency of the results obtained. As well as the rectangular nature of image tiles themselves make the rectangular tiling is a natural choice [2].

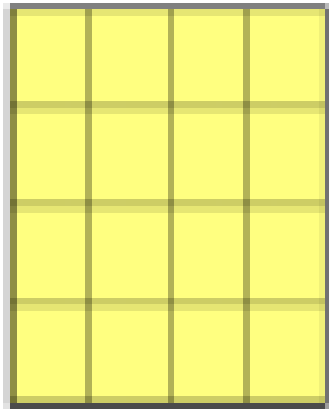

(a)

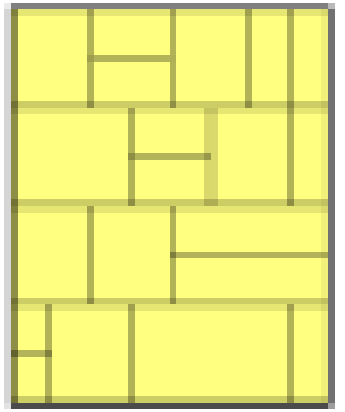

(b)

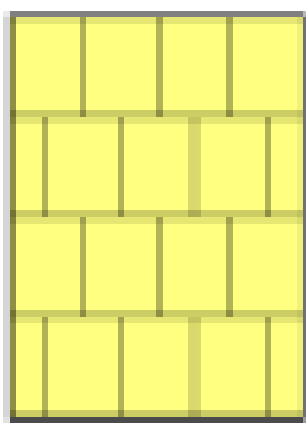

(c)

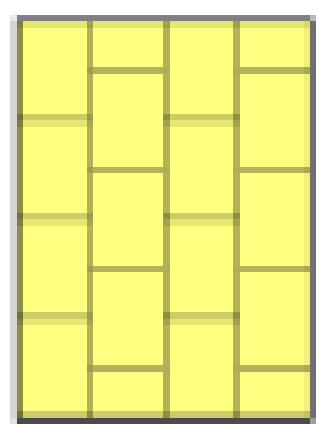

(d)

Figure (2): Tiling pattern.

(a) Regular tiling (b) Irregular tiling

(c) Semi-regular vertical tiling

(d) Semi-regular Horizontal tiling

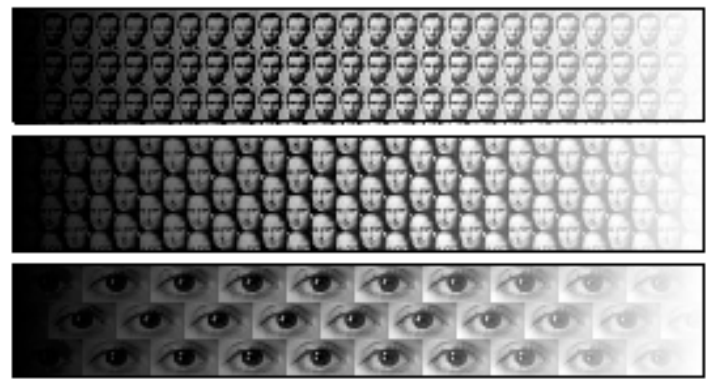

Figure (3): Grayscale image tiles with regular, vertical and horizontal tiling.

\subsection{Find an Arrangement Model of Tiles with in Grid [3][9]}

The arrangement options are:-

a. Uses a unique tile every where, known as periodically mosaic as shown in Figure (4) [2]. 
Creating Image Mosaics using Statistical Methods for Images Alignment.

b. Arrange different tile randomly by eye as shown in Figure (5) [2].

c. Find the same features between target and tile images as shown in Figure (6) [10].
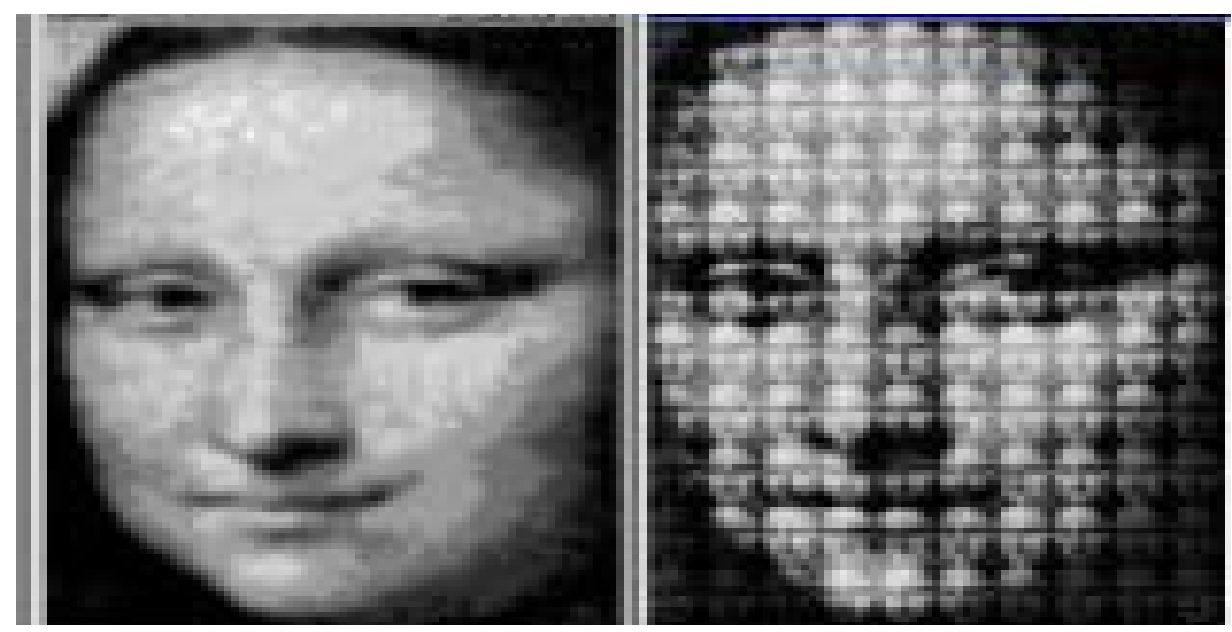

Figure (4): unique tile mosaics

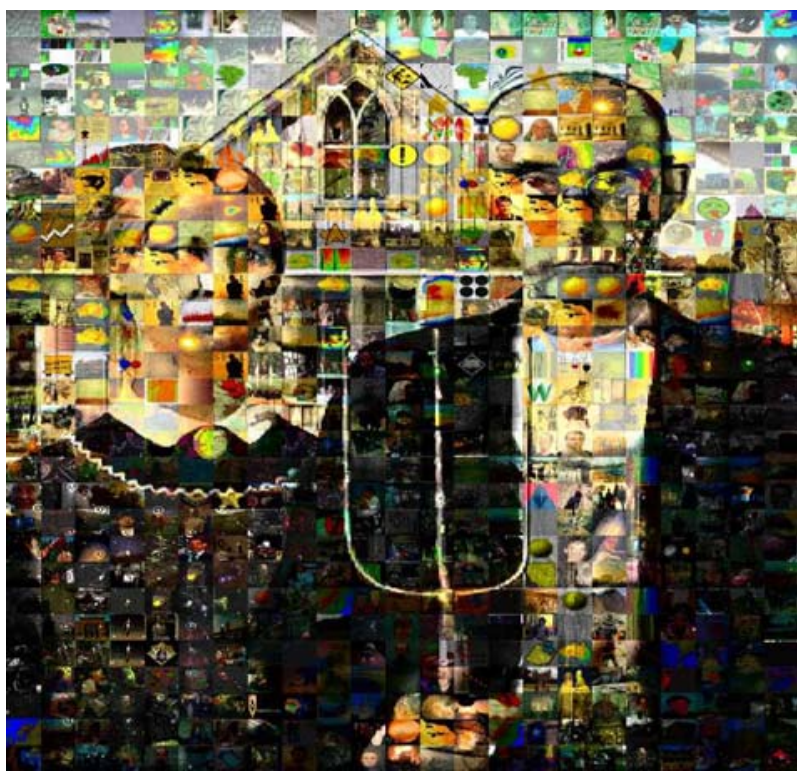

Figure (5): arranging different tiles randomly.
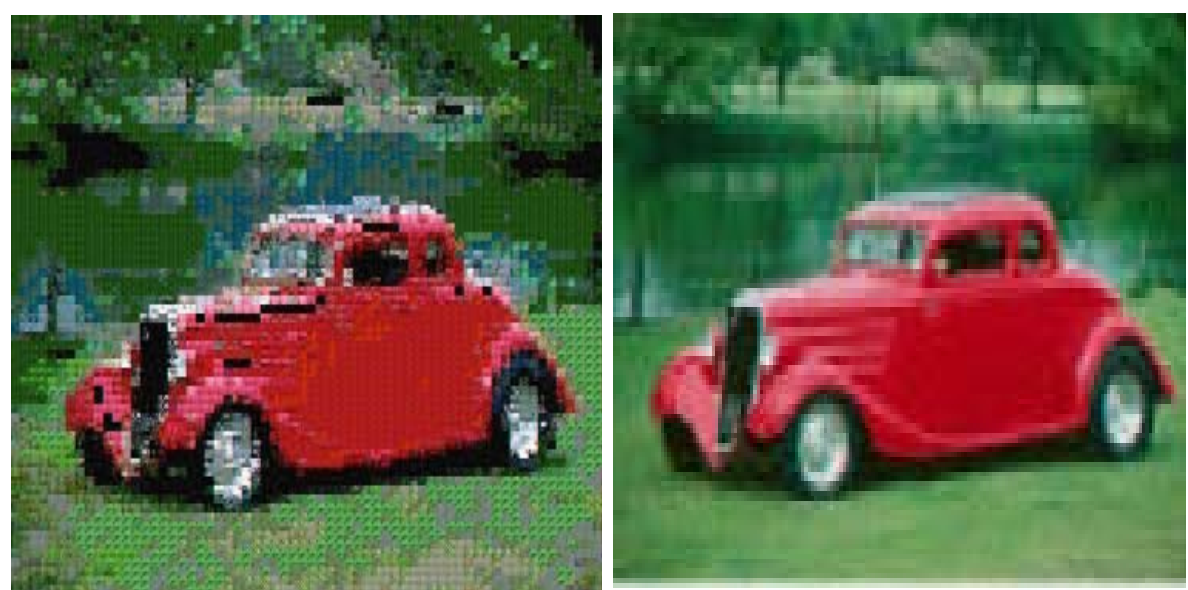

Figure (6): mosaics with same features. 
View correction includes color correction process to adjust the colors of each tile in other words matching the colors between target and tiles images. This correction is obtained through the average approximation between tiles and the target image [3][9].

\section{The Work Flow}

In this section we discuss our work. All the stages are merged together as shown in figure (7). The first two stages to create an image mosaics are performed manually, and are typically iterative. Our work differs from others in that the arranging tile process is performed via the correlation coefficient of intensity.

In order to create an image database thirty colored of different size JPEG (Joint Photograph Expert Group) image is collected. We choose the JPEG format since this format is small in size, simple, easy in manipulation and widely used in visual Databases. The first stage is the use input image, this selection is done manually and randomly a sample from image database is shown in Figure (8).

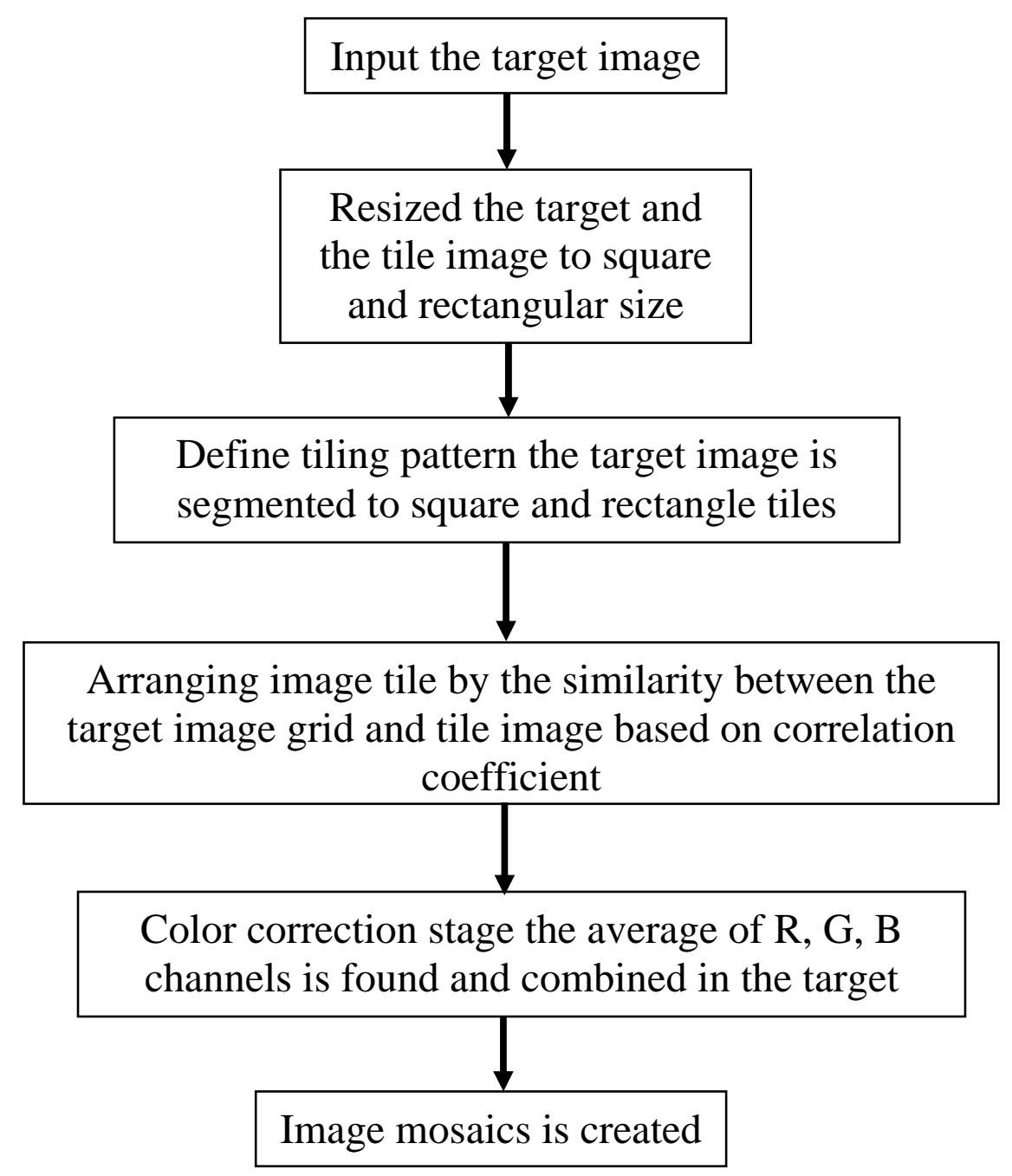

Figure (7): work algorithm. 

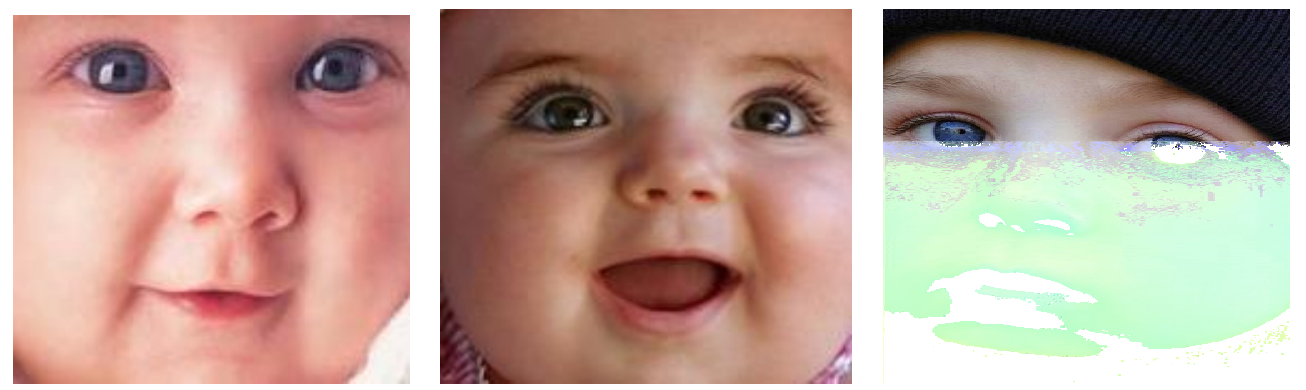

Figure (8): sample from images database.

In order to distinguish the mosaics performed by our work, we resized the target image to a square and a rectangular form of various size that could be shown clearly at the results. Then, we transform all the RGB to a grayscale images to be used in arranging image tiles' stage.

The second stage is selects a tiling pattern. From many tiling patterns shown previously in our system we choose the regular rectangular grids of different size, also we wrenching (تح - توير) the rectangular tile to square grids of $16 \times 16$ pixels. We choose the regular grids for many reasons. The first reason is the simplicity to apply in programming, as we know simplicity is an important factor in programming. The second reason is that the perception of eye is most sensitive to horizontal and vertical grid. Furthermore, the rectangular nature of image tiles themselves make a rectangular tiling is the best choice. These tiles are covered either periodically as one image covered the target image, or non-periodically based on the similarity between the target grid and the tile image.

Arranging the image tiles is the third stage; we need to place the individual image tiles into the target grid. In order to obtain an effective image mosaics the tile images should be similar to the region within the target image. We perform this process with the intensity images via the usage of the correlation coefficient as computed in equation (1) [5]:

$$
\mathbf{C}(\mathbf{x}, \mathbf{y})=\frac{\sum_{\mathbf{i}=1}^{\mathrm{N}}(\mathbf{x i}-\overline{\mathbf{x}})(\mathbf{y i}-\overline{\mathbf{y}})}{\sqrt{\sum_{\mathrm{i}=1}^{\mathrm{N}}(\mathbf{x i}-\overline{\mathbf{x}})^{2} \sum_{\mathbf{i}=1}^{\mathrm{N}}(\mathbf{y i}-\overline{\mathbf{y}})^{2}}}
$$

Where $\bar{x}$ and $\bar{y}$ are mean of tile grid and image tile, $x i$ and $y i$ are pixels of tile grid and image tile. All the tile images are correlated to each grid within the target image. As shown in Figure (9).

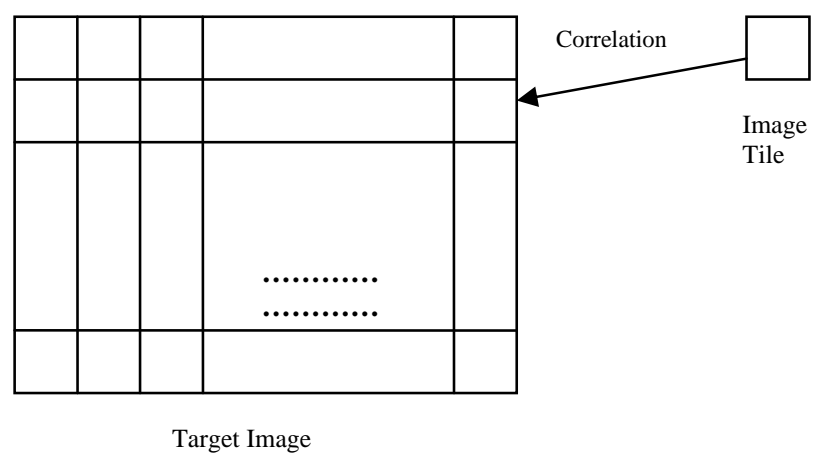

Figure (9): arranging image tile stage. 
The associated thumbnail image with a bigger correlation coefficient between the target grid and the tile image is used to cover the target grid.

The color correction stage, in this stage we adjust the tiles and the target grayscale images. In order to match the target and the tile images we use the process on the original colored tile images as well as the colored target grid. This objective is performed on the three colored channels. We match the colors Red, Green, Blue of the tile image to the color of the region (grid) in the target image that is covered by the tile. All database images are suitable for mosaic from the sense point of view. If the tile image is not suitable from computation point of view the system discard it and other tile image with a bigger correlation coefficient replaced that tile.

This process is done pixel wise, the average of the tile pixels and the target grid pixels is computed according to equation (2) [4]:

$$
\mu_{\mathrm{i}}=\frac{1}{N} \sum_{j=1}^{N} f_{i j}
$$

Where $N$ is the number of the pixels in the color and $f_{i j}$ is the color pixel values. Finally, to perform a color image mosaics this step is necessary and can be omitted. If we omit this step we may end the work with a gray scale mosaics. The three averages of the Red, Green and Blue color channels are returned back and combined in the tile image to cover the target image grid as a layout shown in Figure (10).

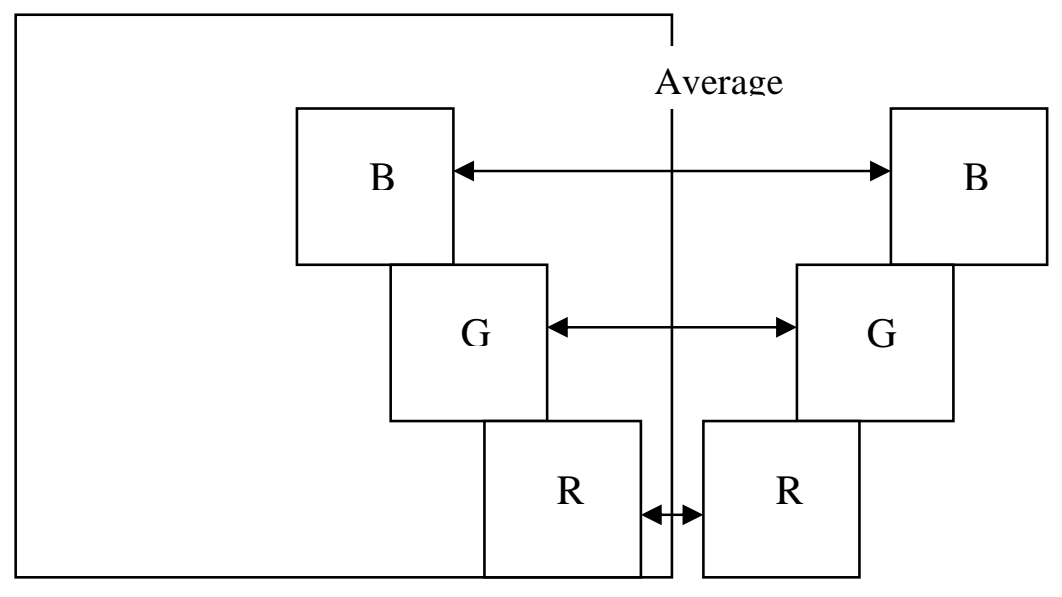

Target image

Tile image

Figure (10): the average of the three colors.

\section{Implementation and Results}

Our work is designed and implemented by MATLAB7 software. We report some examples and quantities results. Figure (11-15) shows a sample from our work results. These results are the image mosaics and its target image. Figure (11) shows the periodically image mosaics of square 


\section{Creating Image Mosaics using Statistical Methods for Images Alignment.}

target and tile images, where the system use one image to cover the target image.

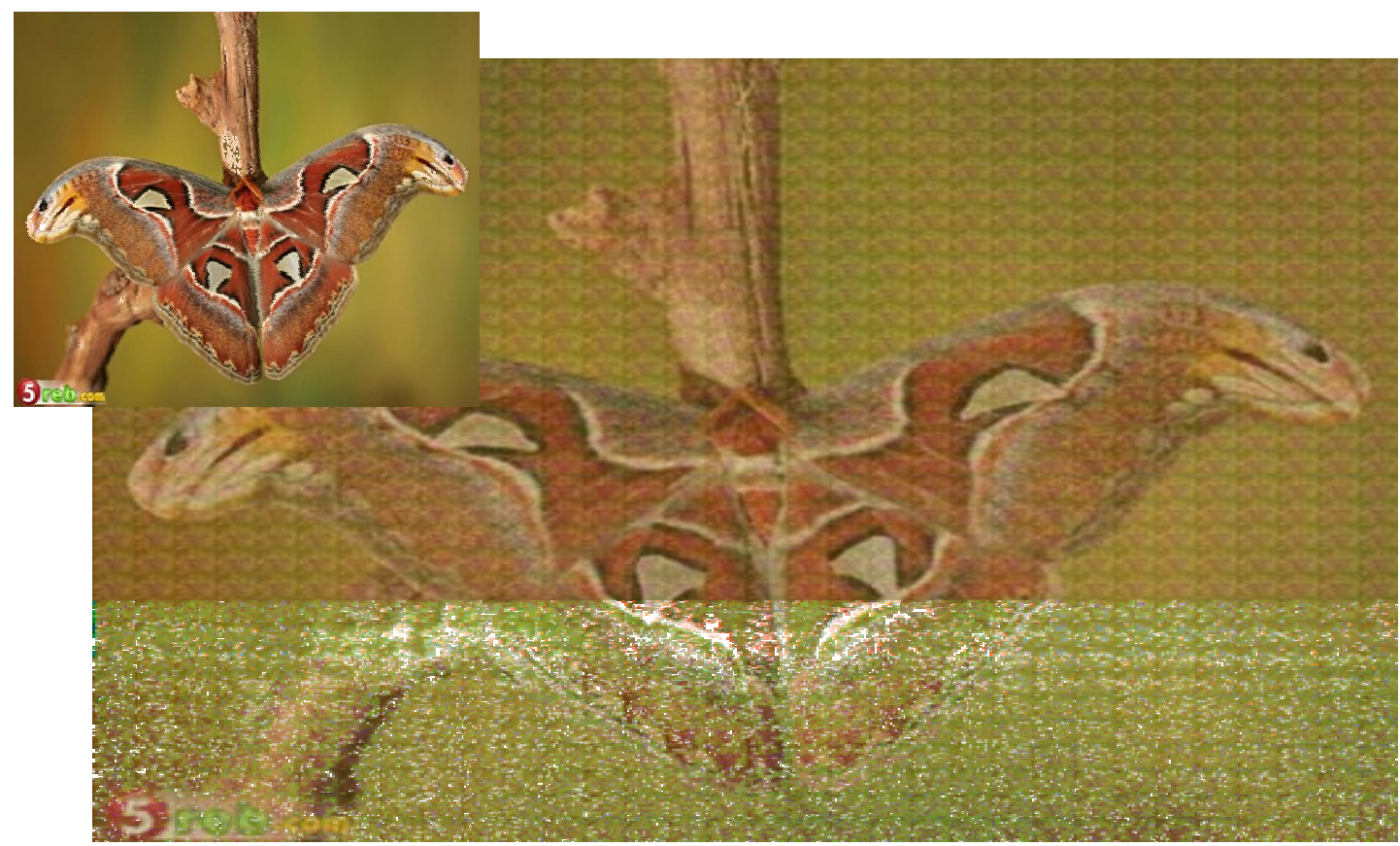

(a)

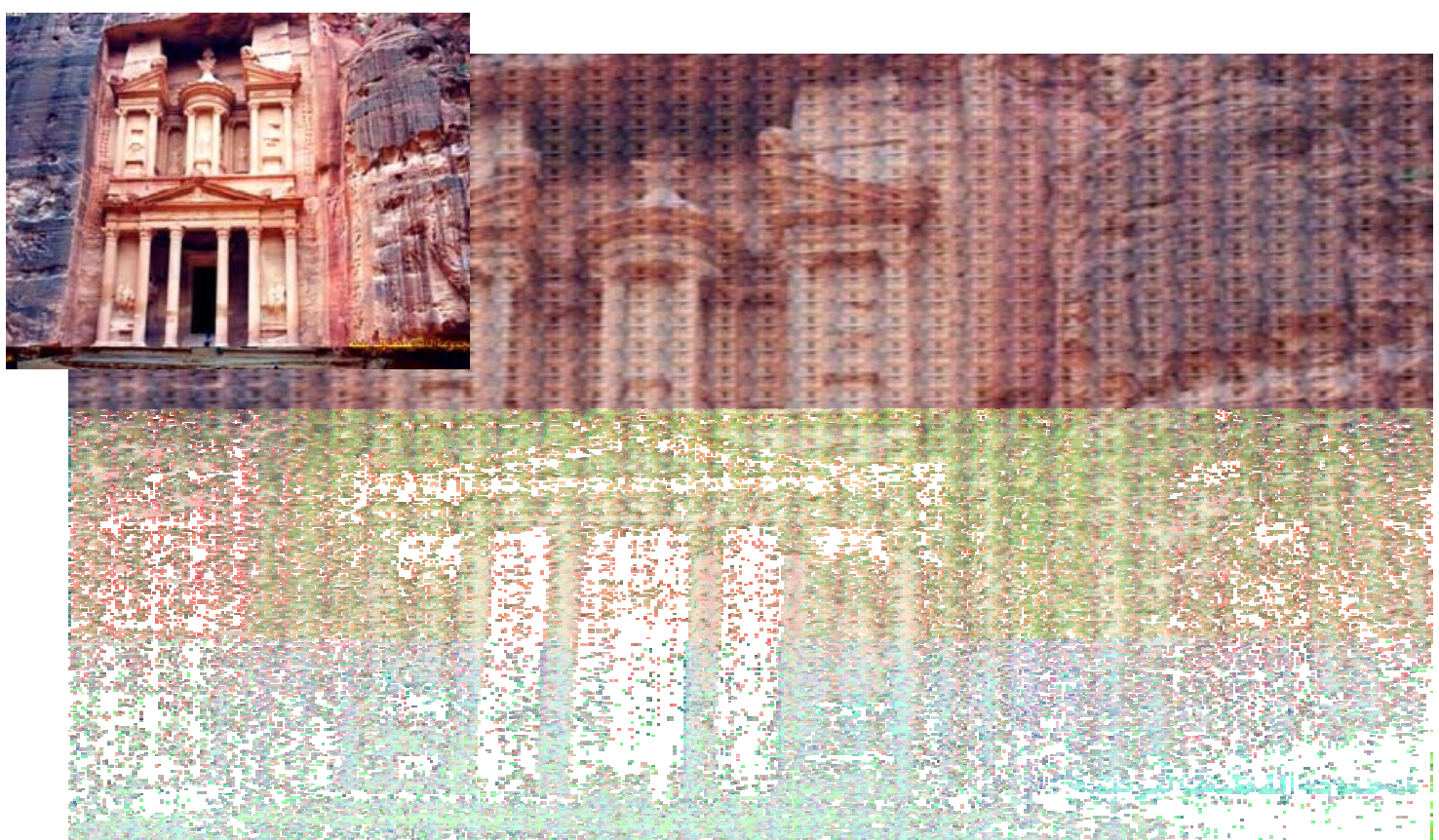

(b)

Figure (11): the work results.

(a) And (b) Periodically Image mosaics , target image on the upper left. Figure (12) shows the non-periodically square image mosaics, where the system use more than one image to cover the target image. 


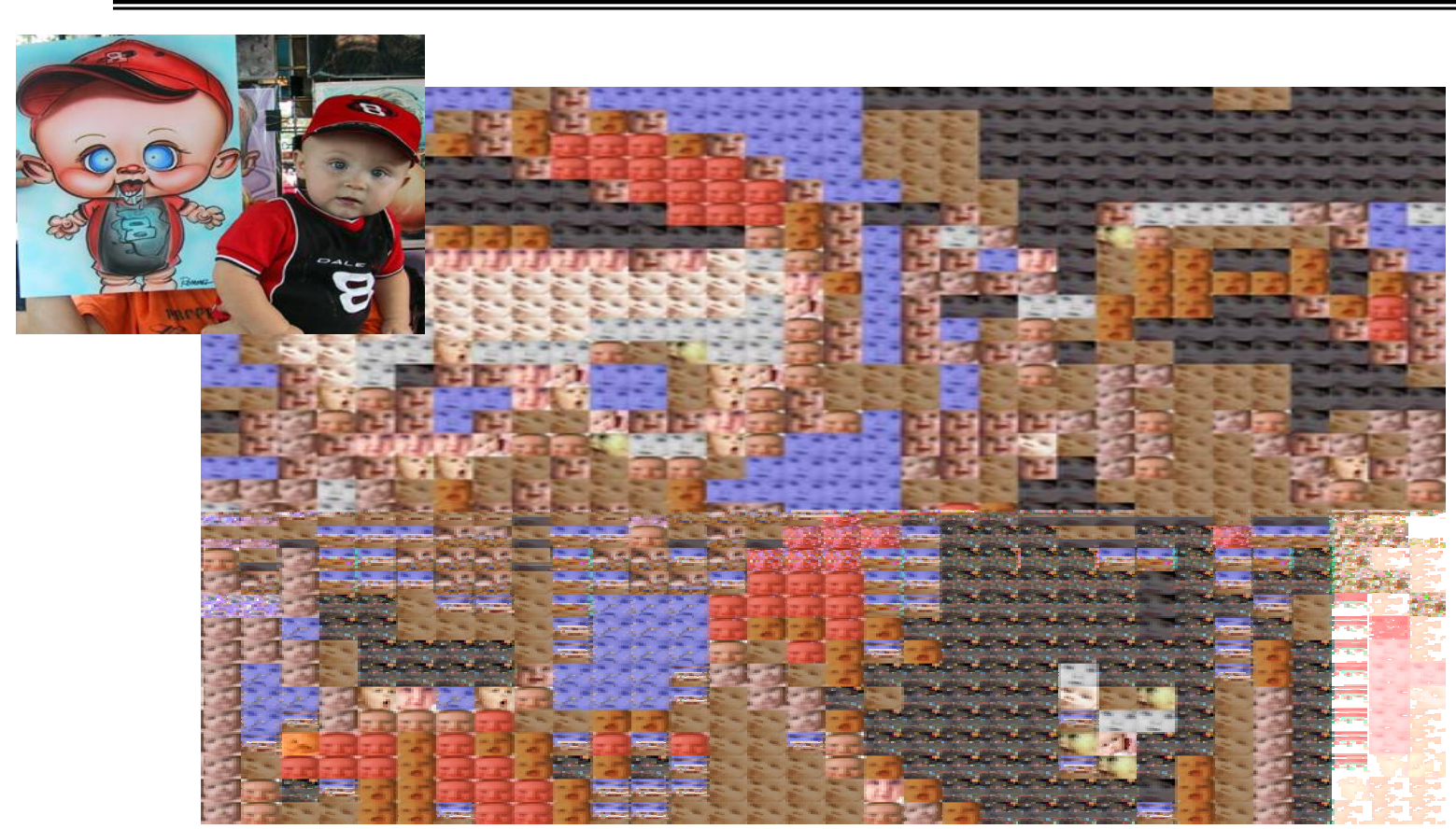

Figure (12): Non-periodically square image mosaics, target image on upper left.

Figure (13) shows the final results of Non-periodically regular rectangular image mosaics of size $608 \times 760$ pixels. The upper left describe the target rectangular image of size 1000x1500 pixels with intermediate mosaics results of 32x38 grids of 19x20 pixel via the system progress.

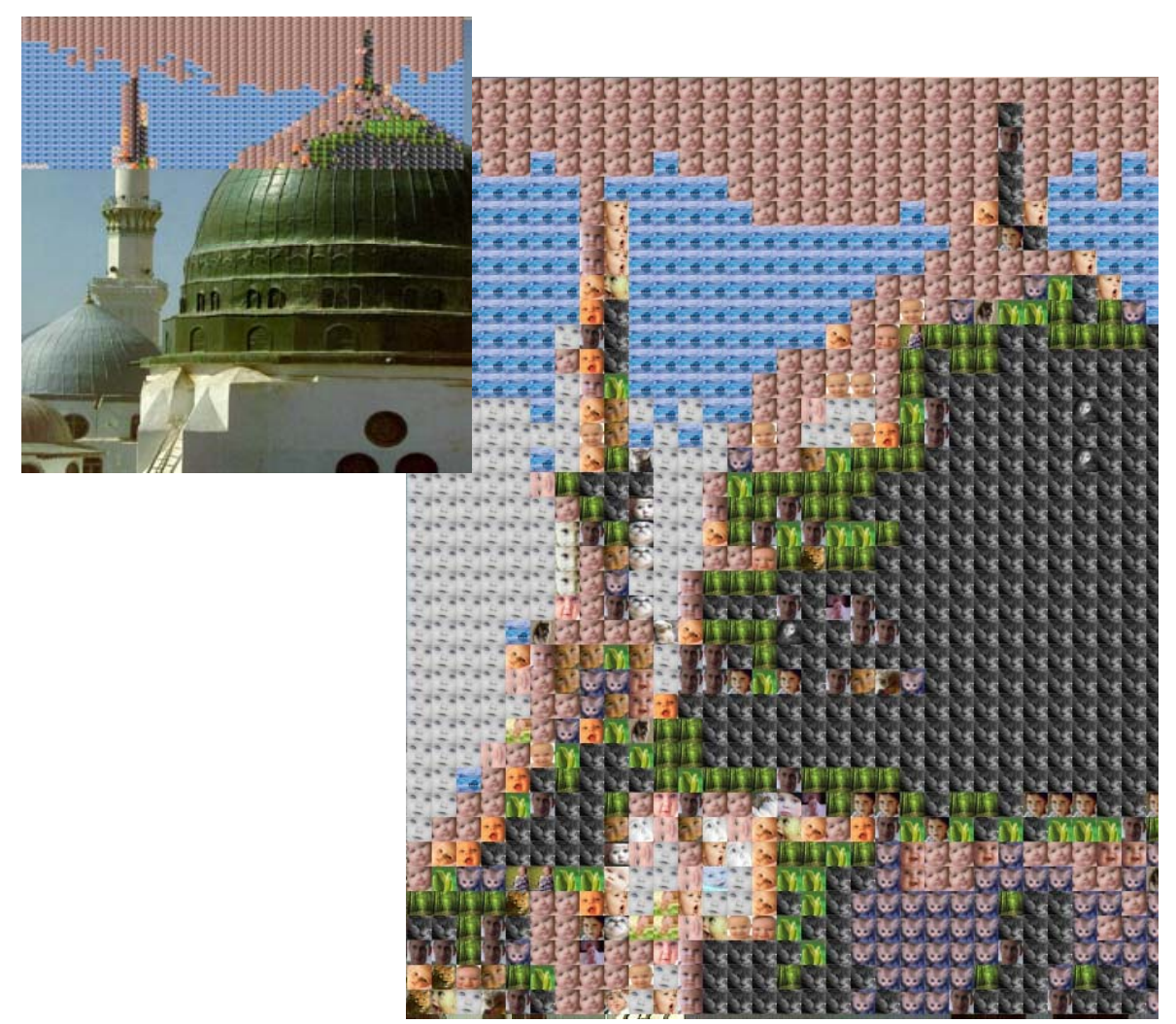

Figure (13) Non-Periodically regular rectangular image mosaics, target image on upper left with intermediate mosaics results 
Figure (14) shows the final results of image size 2000x3000 pixels for target and final results of Non-periodically regular rectangular image mosaics. The upper left describe the target rectangular image with intermediate mosaics results via the system progress.

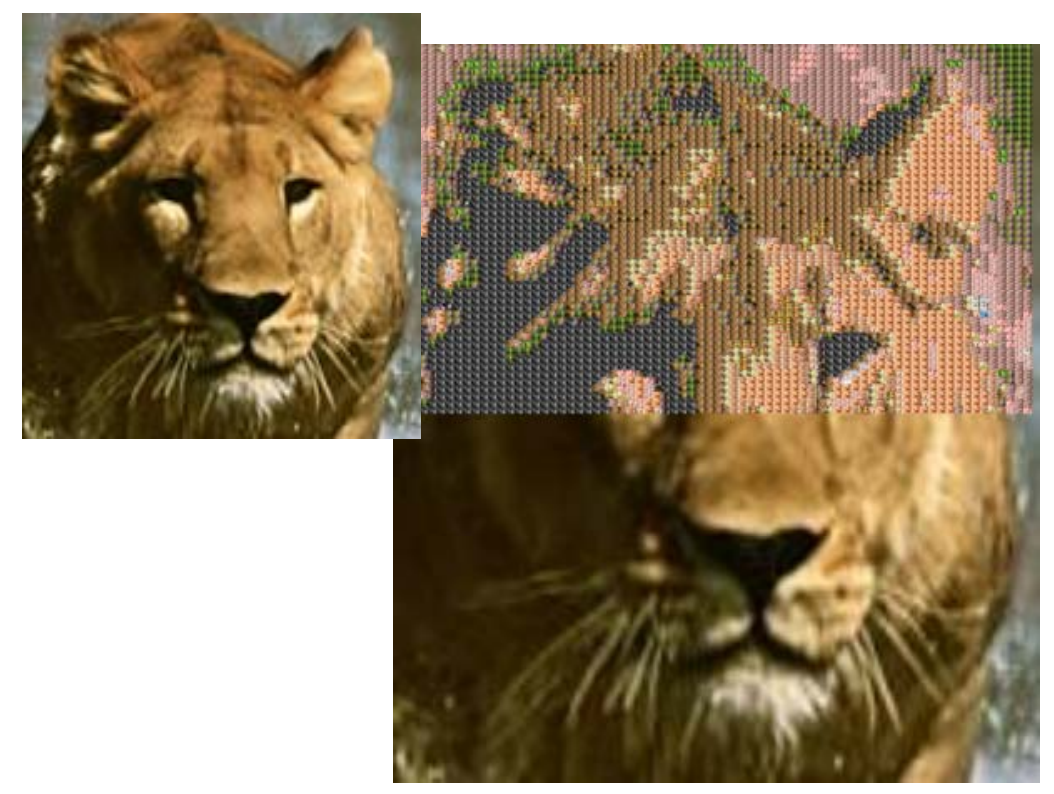

Figure (14): Non-Periodically rectangular image mosaics, target image on upper left

Figure (15) (a) shows the final results for a target original image of size $800 \times 600$ pixels, the final mosaics image of size 300x360 pixel. A regular rectangular image mosaics 20x24 grids per 15x15 pixels each is distributed periodically over the 300x360 pixel image. The upper left describes the target rectangular image the right image shows the mosaics results. Figure (15) (b) shows the final results of image sized 962x1470 pixels for the target image. The tile images is distributed on the final results of non-periodically regular rectangular image mosaics segmented to $74 \times 49$ grids of size $13 \times 30$ pixels. The upper left describe the target rectangular image the right image shows the results of the system.

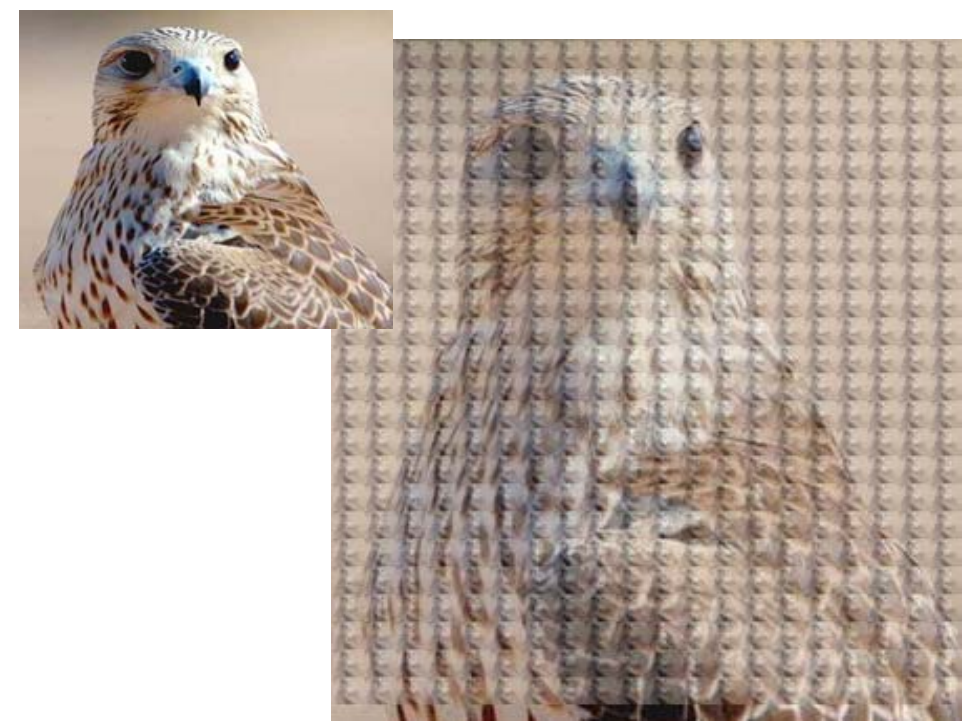

(a) 


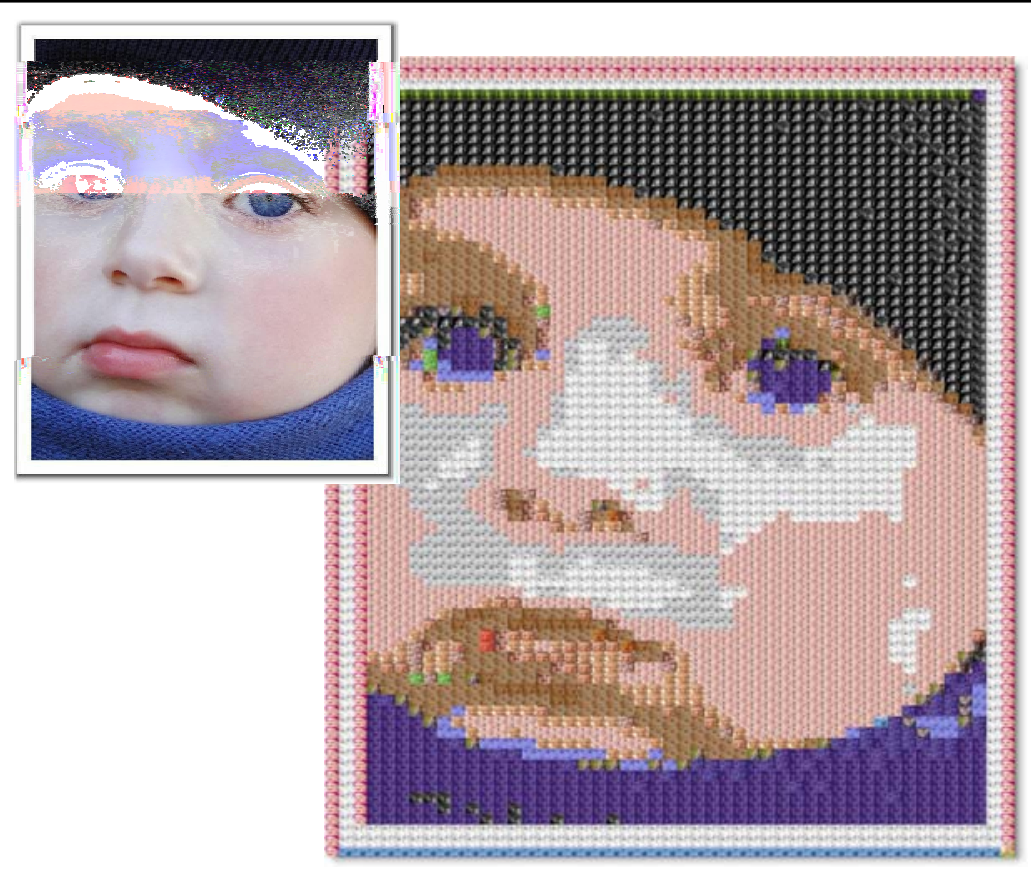

(b)

Figure (15) the work results with the rectangular mosaics

(a) Periodically Image mosaics, target image on upper left (b) Non-periodically Image mosaics, target image on upper left.

The related literature of image mosaics proved that the quality of image mosaic system is difficult to evaluate in a quantitative way. Since there is no appropriate standard measures currently exist to check[1] [10]. Therefore, the comparison of the performance between our system and other systems is completely not fair, despite there aim is similar but there composition approaches are different. So that we evaluate some ideas locally within the system to test its performance. In other word the better the image mosaic depends on the similarity between the image mosaic to its target image. So, in our work, we use the correlation coefficient between an image mosaic represented by tile images and its target image as a criterion to evaluate the mosaic's quality as recorded in table (3). The results obtained by our system are shown in the previous figures. Table (1) records a local comparison of some R, G, B color pixels' values selected randomly between the original target and the original tile images. Depending on the values shown in table (1), the nearest values to the target image are image3 and image4. From table (2) we noted that the nearest intensity pixels' values to the target image are image 3 and image4. Table (3) used the grayscale resized images which proved that the more correlated tile images to the grid determined by the target image are image3 and image4. Image4 is spread over the associated target grid because it produced the larger correlation coefficient than other tile images. 
Creating Image Mosaics using Statistical Methods for Images Alignment.

Table (1): R, G, B pixel values of target and tile images.

\begin{tabular}{|c|c|c|c|c|c|c|}
\hline $\begin{array}{l}\text { Image } \\
\text { name }\end{array}$ & Pixel $(1,1)$ & Pixel(1,2) & $\operatorname{Pixel}(2,1)$ & Pixel(2,2) & Pixel(12,104) & Pixel(100,100) \\
\hline Target & 254, 254, 254 & 254, 254, 254 & 254, 254, 254 & 254, 254, 254 & 202, 202, 202 & 27, 27, 19 \\
\hline Img 1 & 144, 106, 71 & 106, 70, 56 & $108,75,60$ & $83,47,33$ & 118, 70, 66 & 169, 109, 33 \\
\hline Img 2 & 166, 167, 169 & 162, 137, 106 & 167, 143, 109 & 169, 145, 111 & 169, 145, 111 & $162,137,106$ \\
\hline Img 3 & 243, 243, 243 & 239, 232, 224 & 243, 247, 250 & 242, 245, 250 & 243, 247, 91 & 239, 232, 224 \\
\hline Img 4 & 238, 237, 234 & 138, 92, 66 & 237, 228, 233 & 236, 230, 234 & 235, 229, 233 & 138, 192, 66 \\
\hline
\end{tabular}

Table (2): Intensity pixel values of target and tile images.

\begin{tabular}{|c|c|c|c|c|c|c|}
\hline $\begin{array}{c}\text { Image } \\
\text { name }\end{array}$ & Pixel (1,1) & Pixel(1,2) & Pixel(2,1) & Pixel(2,2) & Pixel(12,104) & Pixel(100,100) \\
\hline Target & 255 & 254 & 254 & 254 & 202 & 28 \\
\hline Img 1 & 61 & 79 & 83 & 56 & 84 & 124 \\
\hline Img 2 & 130 & 146 & 148 & 148 & 93 & 141 \\
\hline Img 3 & 231 & 231 & 232 & 231 & 154 & 103 \\
\hline Img 4 & 210 & 246 & 245 & 246 & 132 & 233 \\
\hline
\end{tabular}

Table (3): correlation between target and tile images

\begin{tabular}{|c|c|}
\hline Image name & Correlation \\
\hline Img 1 & .564 \\
\hline Img 2 & .528 \\
\hline Img 3 & .682 \\
\hline Img 4 & .709 \\
\hline
\end{tabular}

\section{Conclusion and Future Work}

We have described a method for creating image mosaics system through a primary and processing operation to the target and image database. The difference between the procedures we proposed than others is that we used the correlation coefficient between the grayscale pixels of the target image as well as the tile images for matching operation. This measure exceeds the others work measures that used the distance function which obtain approximate and not accurate results. 
Future work we will try to use HSV (Hue, Saturation and Value) color space for mosaics. Also, we will try to improve the system to be used for 3D image mosaics. Another idea is to improve the system to be used for image security such encryption and hiding. Indeed, we will try to extend this work to video mosaics.

\section{References}

1) Di Blasi Gianpiero, Gallo Giovanni and Petralia Maria, 2005, "Puzzle Image Mosaic", Proceedings of International Conference on Visualization, Imaging and Image Processing, Benidrom, Spain.

2) Fenkilistine Adam and Range Marisa, 1998, "Image Mosaics", [Chap.] "Electronic Publishing, Artistic Imaging, and Digital Typography", P:11-22, Springer-Verlag: Heidelberg, London, UK.

3) Fenkilistine Adam and Range Marisa, 1998, "Image Mosaics", Presentation Proceedings of EP'98 and RIDT'98 Conference, St. Malo, France.

4) Gonzalez Rafael C. and Richard E. Woods, 2002, "Digital Image Processing". $2^{\text {nd }}$, Prentice-Hall, New Jersey, USA, P:793.

5) Henning Muller, Wolfgang Muller, David McG. Squire and Thierry Pun, 1999, "Performance evaluation in content-based image retrieval: overview and proposals". A Technical Report Vision Submitted to The Computing Science Center, University of Geneva, Geneva, Switzerland.

6) Ren Xuefeng and Tang Guangrong, 2005, "Improving Video Resolution by Image Mosaics", Proceeding of World Academy of Science, Engineering and Technology Vol. 2, Istanbul, Turkey.

7) Shum Heung-Yeung and Szeliski Richard, 1997, "Panoramic Image Mosaics", Technical Report, MSR-TR-97-23 Microsoft Research

8) Szeliski Richard, 1994, "Image Mosaicing for Tele-Reality Applications", Proceedings of the $2^{\text {nd }}$ IEEE workshop, Sarasota, Florida, USA.

9) Thomas Nocke1, Stefan Schlechtweg and Heidrun Schumann, 2005, "Icon-based Visualization using Mosaic Metaphors", Proceedings of IEEE Symposium Information Visualization (IV'05), London, UK.

10) Yue Zhang, 2002, "On the use of CBIR in Image Mosaic Generation", Technical Report 02-17, Submitted to the Department of Computing Science, University of Alberta, Edmonton, Alberta, Canada. 